\title{
Factores relacionados con la calidad del sueño y la somnolencia diurna en universitarios del suroccidente colombiano
}

\section{Related factors with sleep quality and daytime sleepiness in university students from the south-west of Colombia}

\author{
Steven Sánchez-Oviedo (iD) ${ }^{1}$, William Esteban Solarte-Rodríguez (iD) ${ }^{2}$, Sofy Thalía Correa-Carlosama (iD) 3 , Dania Yineth \\ Imbachí-Quinayás (1) 4 , Marlly Lorena Anaya-Ramírez $\mathbb{1 D}^{5}$, Andry Yasmid Mera-Mamián ${ }^{\circledR 6}$, Clara Inés Paz-Peña ${ }^{7}$, Ángela \\ Zúñiga-Pino (1D8
}

1. Universidad del Cauca. Popayán, Colombia. Correo: ssteven@unicauca.edu.co - https://orcid.org/0000-0001-9606-9421

2. Universidad del Cauca. Popayán, Colombia. Correo: esteban95212@unicauca.edu.co - https://orcid.org/0000-0002-1412-3736

3. Universidad del Cauca. Popayán, Colombia. Correo: sofycorrea@unicauca.edu.co - https://orcid.org/0000-0001-6044-9246

4. Universidad del Cauca. Popayán, Colombia. Correo: daniaimbachi@unicauca.edu.co - https://orcid.org/0000-0002-0800-1425

5. Universidad del Cauca. Popayán, Colombia. Correo: marllyan@unicauca.edu.co - https://orcid.org/0000-0002-5717-9617

6. Universidad CES. Medellín, Colombia. Correo: amera@ces.edu.co - https://orcid.org/0000-0002-2356-3370

7. Universidad del Cauca. Popayán, Colombia. Correo: cpaz@unicauca.edu.co - https://orcid.org/0000-0002-3574-3010

8. Universidad del Cauca. Popayán, Colombia. Correo: aezuniga@unicauca.edu.co - https://orcid.org/0000-0002-2264-7265

Tipología: Artículo de investigación científica y tecnológica

Para citar este artículo: Sánchez Oviedo S, Solarte Rodríguez WE, Correa Carlosama ST, Imbachí Quinayás DY, Anaya Ramírez ML, Mera-Mamián A, et al. Factores relacionados con la calidad del sueño y la somnolencia diurna en universitarios del suroccidente colombiano. Duazary. 2021 julio; $18(3)$ : $232-246$. Doi: https://doi.org/10.21676/2389783X.4230

Recibido en junio 12 de 2020

Aceptado en abril 07 de 2021

Publicado en línea en agosto 02 de 2021

Palabras clave: calidad del sueño; somnolencia diurna; rendimiento académico; educación superior.

\section{RESUMEN}

Esta investigación relaciona las características del sueño (calidad y grado de somnolencia) con las características sociodemográficas y académicas, y algunos hábitos de vida. Estudio descriptivo de corte transversal con intención analítica, en el que se aplicaron la escala de somnolencia diurna de Epworth y el índice de calidad del sueño de Pittsburgh a 313 estudiantes de áreas de la salud, mediante cuestionario en línea. El 89,1\% de los participantes se clasificaron como malos dormidores, y el 84,3\% presentaron algún grado de somnolencia diurna, incluida la excesiva (3,5\%). Se encontró relación entre el uso de sustancias para no dormir con la calidad del sueño $(p=0,009)$ y el grado de somnolencia $(p=0,014)$, así como entre el grado de somnolencia diurna y la realización de actividades extracurriculares de tipo laboral $(p=0,029)$. También se dio una correlación débil entre el puntaje de Pittsburg y la edad $(p=0,009)$ y el promedio académico $(p=0,030)$. Se identificó un alto porcentaje de dificultades del sueño y algunos factores asociados, lo que supone la necesidad de educar en la higiene del sueño, dado que investigaciones previas han descrito que estos problemas pueden generar consecuencias negativas como problemas de memoria y concentración.

\section{ABSTRACT}

\section{Keywords:}

Sleep quality; Daytime sleepiness; Academic performance; Health sciences.
This research relates sociodemographic and academic characteristics and some life habits with sleep characteristics (quality and degree of sleepiness). A descriptive cross-sectional study with analytical intention, in which the Epworth daytime sleepiness scale and the Pittsburgh sleep quality index were applied to 313 students in health areas, it was obtained by an online questionnaire. Of the participants, 89.1\% were classified as poor sleepers, $84.3 \%$ had some degree of daytime sleepiness, including excessive daytime sleepiness (3.5\%). A relationship was found between the use of substances for NOT sleeping with sleep quality $(p=0.009)$ and the degree of sleepiness $(p=0.014)$. In the same way, between the degree of daytime sleepiness and the performance of work-related extracurricular activities 
Factores relacionados con la calidad del sueño y la somnolencia diurna en universitarios del suroccidente colombiano

$(p=0.029)$. Also, a weak correlation between the Pittsburg score with age $(p=0.009)$ and academic average $(p=0.030)$. A high percentage of sleep difficulties and some associated factors were identified, which implies the need to educate on sleep hygiene since previous research have described that these can generate long-term negative consequences such as memory and concentration problems.

\section{INTRODUCCIÓN}

El sueño es un proceso fisiológico importante para la salud integral de los seres vivos ${ }^{1-3}$ que, en alternancia con la vigilia, aparece aproximadamente cada 24 horas $^{4,5}$. El tiempo requerido por cada persona para descansar clasifica el sueño en patrones: corto, intermedio y largo, dependiendo de si se descansa cinco horas o menos, siete a ocho horas o más de nueve horas respectivamente ${ }^{4}$. En general, se considera que un promedio de siete a ocho horas de sueño cada 24 horas es lo que necesita un joven para estar despierto y activo durante el día ${ }^{3}$.

Algunas de las funciones del sueño son favorecer la codificación, la memoria y la plasticidad cerebral ${ }^{6}$. Asimismo, su duración influye en aspectos físicos y psicológicos como el estado de ánimo y el bienestar ${ }^{7,8}$. Sin embargo, estos aspectos se ven afectados por condiciones clínicas como la obesidad ${ }^{9}$, emocionales como el estrés y escolares como las demandas académicas ${ }^{10,11}$. También se ha descrito que los trastornos en esta materia pueden estar asociados a mala higiene del sueño ${ }^{12}$.

Los adolescentes, en especial aquellos que estudian en instituciones de educación superior y en programas de ciencias de la salud, tienden a variar sus horarios de sueño. Esto se debe básicamente a factores propios del desarrollo o ambientales como la realización de actividades extracurriculares o de trabajo independiente, acciones que podrían afectar su aprendizaje y el rendimiento académico ${ }^{13}$.

Investigaciones como la de Talero et al. ${ }^{13}$ publicada en 2013 señalan que en los adolescentes tardíos (entre 17 y 20 años) es común el síndrome de fase de sueño retrasada, que dificulta iniciar el descanso nocturno, lo cual a su vez favorece la somnolencia diurna y afecta el funcionamiento normal en las actividades diarias. Las alteraciones de los patrones normales pueden presentarse en duración y en calidad $^{14}$. A largo plazo, los trastornos del sueño desencadenan en algunos individuos patologías como diabetes, hipertensión arterial y enfermedades cerebrovasculares ${ }^{15}$.

La calidad del sueño puede ser evaluada por dominios ${ }^{16}$, y la respuesta positiva o negativa permite clasificar a las personas en buenos o malos dormidores. De esta manera, varias publicaciones realizadas en población universitaria han encontrado prevalencias de malos dormidores superiores al $60 \% \%^{4,5,16}$. Adicionalmente, existen factores externos que pueden favorecer la aparición de estos trastornos. Por ejemplo, se ha identificado que los universitarios que estudian y trabajan tienen menor calidad del sueño ${ }^{5}$.

Otro rasgo por mencionar es la somnolencia, definida como la tendencia de la persona a quedarse dormida, determinada por la calidad y la cantidad del sueño y el ritmo circadiano ${ }^{1,4}$. Esta se clasifica en normal o patológica: la primera somnolencia resulta del ritmo circadiano, y la segunda se da por alteraciones del sueño ${ }^{17,18}$. También existen la somnolencia objetiva, que puede ser evaluada con herramientas como el electroencefalograma, y la subjetiva, relacionada con la percepción de la necesidad de dormir ${ }^{1}$. Una tercera clasificación incluye la somnolencia optativa y la somnolencia diurna excesiva (SDE): la primera tiene que ver con la facilidad de quedarse dormido en un momento socialmente aceptable, mientras que la segunda ocurre cuando el individuo debería estar despierto ${ }^{1,3}$. Esta somnolencia excesiva, en particular, toma gran relevancia en investigaciones de tipo académico, dado que la mayoría de las actividades son desarrolladas durante el día.

En casos de SDE la persona manifiesta un excesivo deseo de dormir en cualquier situac ión ${ }^{19}$, y se caracteriza por pérdida de energía, dificultad para concentrarse y disminución en la memoria ${ }^{1}$. Las causas pueden ser primarias (o de origen central) y secundarias, si hay condiciones subyacentes, por lo cual los hábitos de vida pueden incidir de manera 
Steven Sánchez-Oviedo, William Esteban Solarte-Rodríguez, Sofy Thalía Correa-Carlosama, Dania Yineth Imbachí-Quinayás, Marlly Lorena Anaya-Ramírez, Andry Yasmid Mera-Mamián, Clara Inés Paz-Peña, Ángela Zúñiga-Pino

positiva o negativa sobre la calidad de sueño (consumo de medicamentos, alcohol y tabaquismo o trabajar durante la noche) $)^{1,20,21}$. La SDE se clasifica en: leve, manifestada por alteraciones atencionales y en la concentración; moderada, cuando la somnolencia se presenta principalmente al realizar actividades sedentarias, y severa si el sueño ocurre durante la actividad física ${ }^{4}$.

Asimismo, investigaciones refieren que la falta de sueño se asocia con mayor prevalencia de algunas enfermedades somáticas y trastornos psiquiátricos ${ }^{22}$. Además, al ser el sueño un factor que genera impacto en el funcionamiento físico, psicológico, intelectual y cognoscitivo de una persona, se ha afirmado que puede llegar a influir en aspectos como el rendimiento académico ${ }^{3}$, aunque existe controversia al respecto porque los hallazgos son variados. Algunos autores describen que los trastornos del sueño por sí solos no definen la existencia o no de buen rendimiento académico.

Teniendo en cuenta el impacto que las alteraciones del sueño tienen en el funcionamiento intelectual y cognoscitivo, esta investigación tuvo como objetivo evaluar la relación entre las características del sueño (calidad y grado de somnolencia) y las características sociodemográficas y académicas y algunos hábitos de vida en los estudiantes de la Facultad Ciencias de la Salud de la Universidad del Cauca.

\section{MATERIALES Y MÉTODOS}

\section{Tipo de investigación}

Estudio descriptivo de corte transversal con intención analítica.

\section{Participantes}

La población objetivo fueron estudiantes de los programas de Medicina, Enfermería, Fonoaudiología y Fisioterapia de la Facultad de Ciencias de la Salud de la Universidad del Cauca. Se incluyeron todos aquellos que aceptaron participar mediante la firma del consentimiento informado y que estaban matriculados entre segundo y décimo semestres. Se excluyeron los de primer semestre dado que aún no tenían historial académico para el respectivo análisis, y los de último año de Medicina (once y doceavo semestres) puesto que se encontraban desarrollando sus prácticas clínicas, lo cual podría generar baja participación en encuestas en línea.

Es necesario mencionar que se seleccionó la Facultad de Ciencias de la Salud debido a la filiación de los investigadores con ella, las características específicas de la formación en áreas de la salud y la alta exigencia académica en los cuatro programas de pregrado. Además, es importante considerar la falta de estudios en temas relacionados con el bienestar y el desempeño estudiantil en este ámbito si se tiene en cuenta que son personas que a futuro velarán por el cuidado de otros y requieren buenas condiciones de salud tanto física como mental y emocional.

\section{Instrumento y procedimiento}

Para el proceso de recolección de información se compartió por correo institucional un documento con los objetivos de la investigación y el consentimiento informado. Posterior a ello, se les envió un cuestionario virtual a quienes accedieron a participar (313 estudiantes) a través de la plataforma de Google Forms. Este instrumento constaba de 67 preguntas de selección múltiple en las que se incluyeron variables de caracterización sociodemográfica tales como edad, sexo, estado civil, estrato socioeconómico, etnia, procedencia, pertenencia a grupos especiales, afiliación en salud y número de hijos. De igual forma, se agregaron variables de caracterización académica como programa, semestre, asignaturas perdidas, rendimiento académico antes de ingresar a la universidad, actividades extracurriculares de tipo laboral y rendimiento académico actual. Cabe resaltar que el promedio académico del semestre inmediatamente anterior se presenta como una variable cuantitativa, y a partir de este dato se realizó la medición de la variable rendimiento académico, clasificándolo en alto (igual o mayor a $4,0)$, medio $(3,5$ a 3,9$)$, regular $(3,0$ a 3,4$)$ y bajo (menor a 3,0). También se incluyó información sobre hábitos de práctica de actividad física y consumo de cigarrillo y de alcohol ${ }^{16}$. 
La variable calidad del sueño (global) se definió según el índice de calidad del sueño de Pittsburgh (ICSP), el cual ha sido validado para Colombia por Escobar-Córdoba y Eslava-Schmalbach ${ }^{23}$, de la Facultad de Medicina de la Universidad Nacional de Colombia. La escala consta de 19 preguntas y se puntúa en un rango de 0 a 21 . A mayor puntaje, menor calidad subjetiva del sueño $0^{5,16}$, de manera que un puntaje menor a cinco indica buena calidad del sueño, y un puntaje igual o mayor de cinco refleja mala calidad de sueño ${ }^{20}$. Esta herramienta permite identificar además los dominios calidad subjetiva del sueño, latencia de sueño, duración del sueño, eficiencia habitual del sueño, perturbaciones extrínsecas del sueño, uso de medicación hipnótica y disfunción diurna ${ }^{16}$.

Por otra parte, la variable somnolencia diurna permitió clasificar a los estudiantes sin somnolencia diurna (0-6 puntos), con ligera somnolencia diurna (7-13 puntos), de moderada somnolencia diurna (14-19 puntos) y con somnolencia diurna excesiva (SDE) (20-24 puntos). Para ello se consideraron las preguntas relacionadas con la tendencia a quedarse dormido mientras se está sentado o leyendo, viendo televisión, sentado sin actividad, como copiloto de un viaje, recostado a media tarde, sentado y charlando con alguien, sentado después de la comida, conduciendo en su carro y cuando se para durante algunos minutos debido al tráfico ${ }^{24}$.

\section{Análisis de datos}

El procesamiento de la información se realizó mediante el programa Jamovi ${ }^{\circledR 25,26}$. Las variables cualitativas fueron analizadas mediante frecuencias y porcentajes, mientras que las cuantitativas se presentan a través de medidas de tendencia central y de dispersión, según la distribución de los datos. En el análisis bivariado se relacionaron las variables calidad del sueño (buena/mala) y grado de somnolencia diurna (sin somnolencia/ligera o moderada/excesiva) con variables sociodemográficas y académicas utilizando las pruebas chi cuadrado, la prueba exacta de Fisher, $\mathrm{U}$ de Mann-Whitney y t de Student para muestras independientes, según las características de los datos. También se evaluó la relación entre la edad (años) y el promedio académico (valor numérico del semestre inmediatamente anterior) utilizando la correlación de Pearson. Cabe mencionar que para todos los análisis se consideró un nivel de significancia de 0,05.

\section{Declaración sobre aspectos éticos}

Se adoptaron las normas establecidas en la Declaración de Helsinki de 1975 y la Resolución 8430 de 1993 del Ministerio de Salud de Colombia. Solo se incluyeron estudiantes que aprobaron su participación a través de la aceptación del consentimiento informado. Se contó con el aval ético de la Universidad del Cauca, bajo Resolución 4748 de la Vicerrectoría de Investigaciones.

\section{RESULTADOS}

Tras realizar un piloto aplicando la encuesta de caracterización social, demográfica y académica a 36 participantes del programa de Fisioterapia que tenían matriculadas asignaturas prácticas y teóricas, se decidió eliminar algunas preguntas que no se consideraron de utilidad para los objetivos de la investigación. El tiempo de respuesta promedio fue de 20 minutos.

\section{Características sociodemográficas}

Participaron un total de 313 estudiantes con una mediana de edad de 21 años (RIC: 20-23). El más joven tenía 17 años, y el mayor, 33. El promedio de edad fue de $21,9 \pm 2,72$ años. Del total de participantes, la mayoría eran mujeres $(71,2 \%)$, solteros $(95,2 \%)$ y pertenecían al estrato bajo, niveles I y II (62,9\%). El $86,6 \%$ se consideran mestizos, y un $8,0 \%$ se identificaron como indígenas. El mayor porcentaje procede del departamento del Cauca (62,3\%), seguido por los departamentos de Nariño (19,5\%), Huila $(5,4 \%)$ y Valle $(5,1 \%)$. Respecto al sistema de salud, se obtuvo el dato para 311 de los universitarios, de los cuales el 96,8\% (301) manifestaron estar afiliados a una empresa prestadora de salud (EPS).

\section{Características académicas}

Del total de participantes el 34,2\% pertenecían al programa de Fonoaudiología; 29,7\%, a Medicina; 
23,6\%, a Fisioterapia, y 12,5\%, a Enfermería. La mayor participación fue de cuarto semestre $(21,7 \%)$. Por otro lado, el 54,3\% refirieron haber perdido asignaturas en el programa que cursaban, siendo segundo semestre el que presentó mayor número de asignaturas perdidas (36\%). También se identificó que el $16,9 \%$ de los estudiantes incluían actividades extracurriculares de tipo laboral. En cuanto al rendimiento académico de los 313 encuestados, el $24,9 \%$ tenían un desempeño alto; el $55,9 \%$, medio; $14,7 \%$, regular, y $4,5 \%$, malo.

\section{Hábitos}

El 57,5\% de los participantes (180) manifestaron practicar actividad física. De estos, 177 registraron la frecuencia, y la mayoría $(57,1 \%)$ la realizan entre una y tres veces por semana. El resto (43\%) la practican cuatro veces o más. En relación con el cigarrillo y el alcohol, $23(7,35 \%)$ y $92(29,4 \%)$ participantes refirieron consumirlos, respectivamente, al menos una vez por semana.

Respecto a sustancias para no dormir, el 61,7\% (193) afirmó utilizarlas, siendo el café la bebida más frecuente (187/193). Además, se identificó que en este grupo algunos ingerían de manera simultánea café y energizantes (72/193). Otras de las sustancias consumidas en menor porcentaje incluyeron aromáticas, azúcar, agua y bebidas gaseosas oscuras.

El 5,8\% de los participantes mencionaron utilizar productos para conciliar el sueño. En este caso predominó el uso de medicamentos como antihistamínicos, hipnóticos, y relajantes musculares en su mayoría automedicados. Incluso expresaron ingerir leche, aromáticas y fumar tabaco.

\section{Características del sueño}

Al evaluar la calidad del sueño según sus componentes, se encontró que el $60,7 \%$ la calificaron como "bastante mala o muy mala". El $41,5 \%$ tarda entre 16 y 30 minutos en quedarse dormidos (latencia), y la mayoría $(58,8 \%)$ refirió dormir entre cinco y seis horas; solo el 6,4\% indicó dormir más de siete horas. El 87,2\% manifestó tener un sueño eficiente, es decir, duermen más del $85 \%$ del tiempo que permanecen acostados. De igual forma, el 96,2\% (301) de los participantes reportaron algún tipo de perturbación como: despertarse durante la noche o de madrugada, no poder respirar bien, toser o roncar ruidosamente, tener "malos sueños" o pesadillas. Respecto al componente de medicación en el último mes, la mayoría 93\% (291) no registró uso de esta. Además, el 86,9\% (272) expresó dificultades para mantenerse despierto o falta de ánimo al realizar actividades como conducir, comer, trabajar, estudiar o alguna actividad social. Finalmente, el $89,1 \%$ (279) se clasificó como mal dormidor, y el $10,9 \%$ (34) se consideró buen dormidor (tabla 1).

Respecto a la somnolencia diurna, el 84,3\% (264) la presentó, y de estos el 4,17\% (11/264) se clasificó con somnolencia diurna excesiva (figura 1 ).

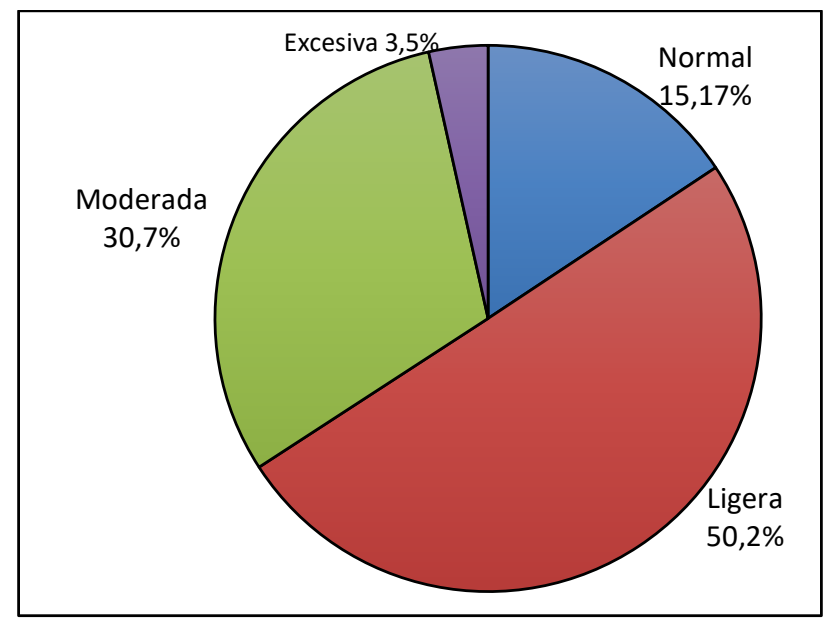

Figura 1. Grado de somnolencia diurna de los estudiantes. 
Tabla 1. Calidad del sueño de los estudiantes.

\begin{tabular}{|c|c|c|c|}
\hline \multicolumn{2}{|l|}{ Dominios calidad del sueño } & \multirow{2}{*}{$\begin{array}{l}n \\
20\end{array}$} & \multirow{2}{*}{$\begin{array}{l}\% \\
6,4\end{array}$} \\
\hline Calidad subjetiva & Muy buena & & \\
\hline & Bastante buena & 103 & 32,9 \\
\hline & Bastante mala & 156 & 49,8 \\
\hline & Muy mala & 34 & 10,9 \\
\hline \multirow{4}{*}{$\begin{array}{l}\text { Latencia } \\
\text { (Minutos que tarda en quedarse dormido) }\end{array}$} & Menos de 15 & 84 & 26,8 \\
\hline & Entre 16 y 30 & 130 & 41,5 \\
\hline & Entre 31 y 60 & 72 & 23 \\
\hline & Más de 60 & 27 & 8,6 \\
\hline \multirow[t]{4}{*}{ Duración del sueño (horas) } & Más de 7 & 20 & 6,4 \\
\hline & Entre 6 y 7 & 42 & 13,4 \\
\hline & Entre 5 y 6 & 184 & 58,8 \\
\hline & Menos de 5 & 67 & 21,4 \\
\hline \multirow{4}{*}{$\begin{array}{l}\text { Eficiencia } \\
\text { (tiempo que realmente duerme estando acostado) }\end{array}$} & Más del 85\% & 273 & 87,2 \\
\hline & Entre el $75-84 \%$ & 24 & 7,7 \\
\hline & Entre $65-74 \%$ & 9 & 2,9 \\
\hline & Menos del 65\% & 7 & 2,2 \\
\hline \multirow{4}{*}{$\begin{array}{l}\text { Perturbaciones } \\
\text { Puntaje entre 0-27 (peor) }\end{array}$} & Puntaje 0 & 12 & 3,8 \\
\hline & Entre 1 y 9 & 226 & 72,2 \\
\hline & Entre 10 y 18 & 69 & 22 \\
\hline & Entre 19 y 27 & 6 & 1,9 \\
\hline \multirow[t]{4}{*}{ Medicación en el último mes } & Ninguna vez en el último mes & 291 & 93 \\
\hline & Menos de una vez a la semana & 12 & 3,8 \\
\hline & Una o dos veces a la semana & 8 & 2,6 \\
\hline & Tres o más veces a la semana & 2 & 0,6 \\
\hline \multirow{4}{*}{$\begin{array}{l}\text { Disfunción diurna } \\
\text { Puntaje entre } 0 \text { y } 6 \text { (peor) }\end{array}$} & Puntaje 0 & 41 & 13,1 \\
\hline & Entre 1 y 2 & 132 & 42,2 \\
\hline & Entre 3 y 4 & 114 & 36,4 \\
\hline & Entre 5 y 6 & 26 & 8,3 \\
\hline \multirow[t]{2}{*}{ Calidad del sueño } & Buenos dormidores & 34 & 10,9 \\
\hline & Malos dormidores & 279 & 89,1 \\
\hline
\end{tabular}

Calidad del sueño según características sociodemográficas, académicas y hábitos de vida

Se halló una relación estadísticamente significativa entre la calidad del sueño y el uso de sustancias para no dormir $(p=0,009)$. La mayoría de las personas con mala calidad del sueño recurren a estos elementos (tabla 2).

\section{Somnolencia diurna según características sociodemográficas, académicas y hábitos de vida}

Se encontró relación estadísticamente significativa entre la somnolencia diurna y las variables realización de actividades extracurriculares de tipo laboral $(p=0,029)$ y uso de sustancias para no dormir $(p=0,014)$. La mayoría de las personas con somnolencia diurna moderada o severa no incluyen actividades laborales extracurriculares y usan sustancias para no dormir (tabla 3 ). 
Steven Sánchez-Oviedo, William Esteban Solarte-Rodríguez, Sofy Thalía Correa-Carlosama, Dania Yineth Imbachí-Quinayás, Marlly Lorena Anaya-Ramírez, Andry Yasmid Mera-Mamián, Clara Inés Paz-Peña, Ángela Zúñiga-Pino

Tabla 2. Calidad del sueño según características sociodemográficas, académicas y hábitos de vida.

\begin{tabular}{|c|c|c|c|c|c|}
\hline \multirow{2}{*}{\multicolumn{2}{|c|}{ Variable }} & \multirow{3}{*}{$\begin{array}{r}\begin{array}{r}\text { Total } \\
\text { n (\%) }\end{array} \\
223(71,2) \\
\end{array}$} & \multicolumn{2}{|c|}{$\begin{array}{c}\text { Calidad del sueño } \\
n(\%)\end{array}$} & \multirow{3}{*}{$\begin{array}{r}\mathbf{p} \\
0,130 \\
\end{array}$} \\
\hline & & & Buena & Mala & \\
\hline \multirow[t]{2}{*}{ Sexo } & Mujer & & $28(82,4)$ & $195(69,9)$ & \\
\hline & Hombre & $90(28,8)$ & $6(17,6)$ & $84(30,1)$ & \\
\hline Edad en años & Mediana (rango) & $21(17-33)$ & $21(17-27)$ & $21(17-33)$ & $0,280^{\mathrm{U}}$ \\
\hline \multirow[t]{2}{*}{ Estado civil } & Soltero/separado & $13(4,2)$ & $0(0)$ & $13(4,7)$ & $0,374^{\mathrm{F}}$ \\
\hline & Casado/unión libre & $300(95,8)$ & $34(100)$ & $266(95,3)$ & \\
\hline \multirow{2}{*}{$\begin{array}{l}\text { Estrato } \\
\text { socioeconómico }\end{array}$} & Bajo (nivel I y II) & $197(62,9)$ & $18(52,9)$ & $179(64,2)$ & 0,201 \\
\hline & Medio (nivel III-VI) & $116(37,1)$ & $16(47,1)$ & $100(35,8)$ & \\
\hline \multirow[t]{2}{*}{ Grupos especiales } & Víctima del conflicto & $22(7)$ & $4(11,8)$ & $18(6,5)$ & $0,278^{\mathrm{F}}$ \\
\hline & Ninguno & $291(93)$ & $30(88,2)$ & $261(93,5)$ & \\
\hline \multirow[t]{4}{*}{ Programa } & Fonoaudiología & $107(34,2)$ & $16(47,1)$ & $91(32,6)$ & 0,236 \\
\hline & Medicina & $93(29,7)$ & $6(17,6)$ & $87(31,2)$ & \\
\hline & Fisioterapia & $74(23,6)$ & $9(26,5)$ & $65(23,3)$ & \\
\hline & Enfermería & $39(12,5)$ & $3(8,8)$ & $36(12,9)$ & \\
\hline \multirow{2}{*}{$\begin{array}{l}\text { Pérdida de al menos } \\
\text { una asignatura }\end{array}$} & Sí & $170(54,3)$ & $18(52,9)$ & $152(54,5)$ & 0,865 \\
\hline & No & $143(45,7)$ & $16(47,1)$ & $127(45,5)$ & \\
\hline \multirow{2}{*}{$\begin{array}{l}\text { Realiza actividades } \\
\text { extracurriculares de } \\
\text { tipo laboral }\end{array}$} & Sí & $53(16,9)$ & $4(11,8)$ & $49(17,6)$ & 0,477 \\
\hline & No & $260(83,1)$ & $30(88,2)$ & $230(82,4)$ & \\
\hline Promedio académico & Media (DE) & $3,70(0,379)$ & $3,66(0,370)$ & $3,71(0,380)$ & $0,229^{\top}$ \\
\hline \multirow{2}{*}{$\begin{array}{l}\text { Realización de actividad } \\
\text { física }\end{array}$} & Sí & $180(57,5)$ & $19(55,9)$ & $161(57,7)$ & 0,839 \\
\hline & No & $133(42,5)$ & $15(44,1)$ & $118(42,3)$ & \\
\hline \multirow[t]{2}{*}{ Consumo de cigarrillo } & Sí & $23(7,3)$ & $1(2,9)$ & $22(7,9)$ & $0,489^{\mathrm{F}}$ \\
\hline & No & $290(92,7)$ & $33(97,1)$ & $257(92,1)$ & \\
\hline \multirow[t]{2}{*}{ Consumo de alcohol } & Sí & $92(29,4)$ & $9(26,5)$ & $83(29,7)$ & 0,692 \\
\hline & No & $221(70,6)$ & $25(73,5)$ & $196(70,3)$ & \\
\hline \multirow{2}{*}{$\begin{array}{l}\text { Uso de sustancias para } \\
\text { no dormir }\end{array}$} & Sí & $193(61,7)$ & $14(41,2)$ & $179(64,2)$ & $0,009 *$ \\
\hline & No & $120(38,3)$ & $20(58,8)$ & $100(35,8)$ & \\
\hline \multirow{2}{*}{$\begin{array}{l}\text { Uso de productos para } \\
\text { conciliar el sueño }\end{array}$} & Sí & $18(5,8)$ & $0(0)$ & $18(6,5)$ & $0,236^{\mathrm{F}}$ \\
\hline & No & $295(94,2)$ & $34(100)$ & $261(93,5)$ & \\
\hline
\end{tabular}

${ }^{*}$ Resultado estadísticamente significativo, con $\mathrm{p}<0,05$. U: prueba U de Mann-Whitney. DE: desviación estándar. T: t de Student para muestras independientes. F: prueba exacta de Fisher 
Tabla 3. Grado de somnolencia diurna según características sociodemográficas, académicas y hábitos de vida.

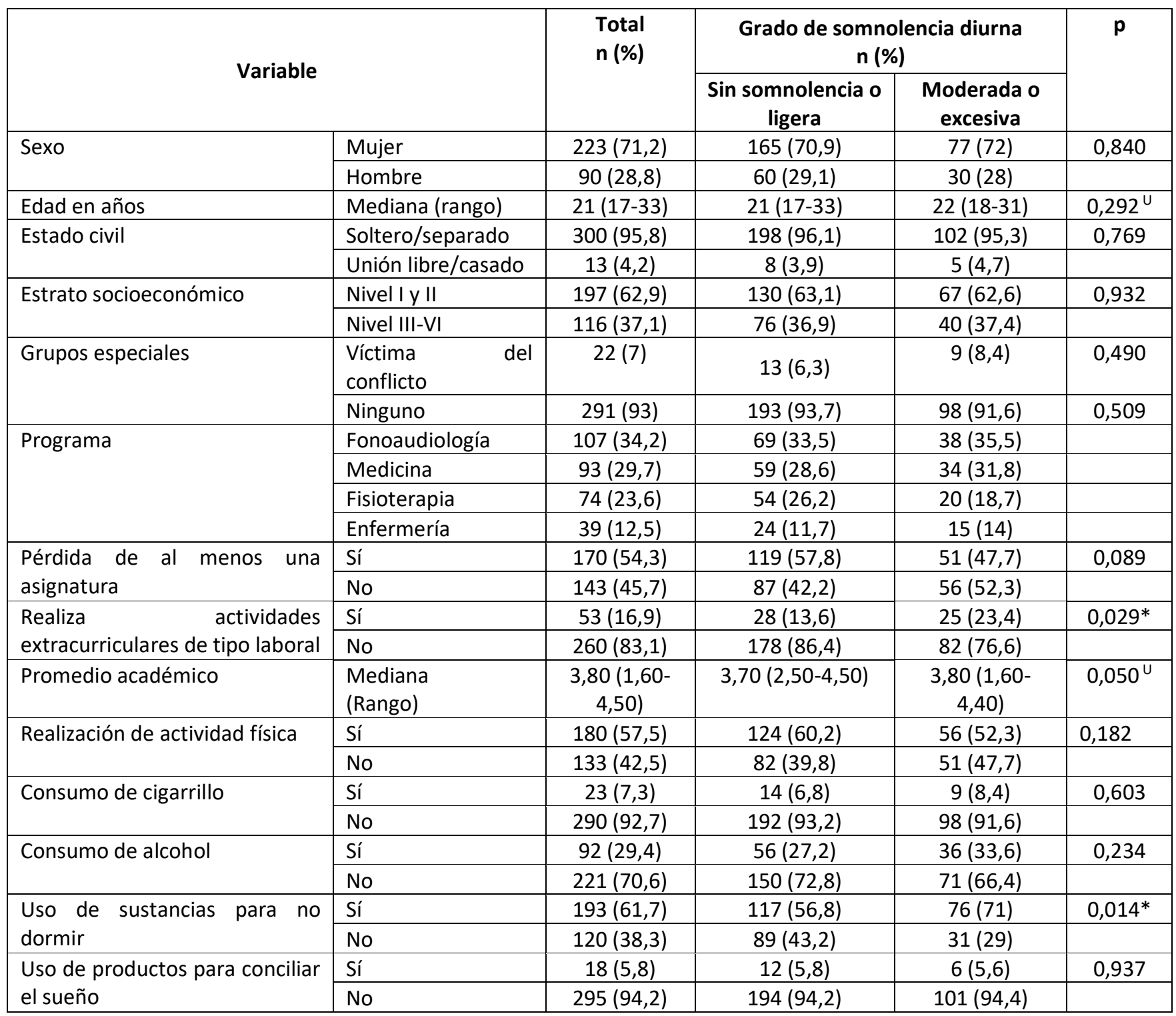

*Resultado estadísticamente significativo, con $\mathrm{p}<0,05$. U: Prueba U de Mann-Whitney.

Tabla 4. Correlación entre la edad y el promedio académico con los puntajes de la escala de calidad del sueño de Pittsburg y de somnolencia diurna de Epworth.

\begin{tabular}{|c|c|c|}
\hline Variable & $\begin{array}{c}\text { Puntaje de calidad del sueño } \\
\mathbf{R}^{2}(\text { IC95\%) }\end{array}$ & $\begin{array}{c}\text { Puntaje somnolencia diurna } \\
\mathbf{R}^{2} \text { (IC95\%) }\end{array}$ \\
\hline Edad en años & $0,147(0,037-0,254), p=0,009^{*}$ & $0,047(-0,065-0,157), p=0,411$ \\
\hline Promedio académico & $-0,122(-0,012-(-0,230)), p=0,030^{*}$ & $0,027(-0,084-0,138), p=0,631$ \\
\hline
\end{tabular}




\section{Correlación entre la edad y el promedio académico con las variables de sueño}

Al realizar la exploración entre los puntajes de calidad del sueño y grado de somnolencia diurna, se identificó correlación positiva débil entre la calidad de sueño y la edad $(p=0,009)$ y correlación negativa débil entre el promedio académico y la calidad del sueño $(p=0,030)$. A mayor edad, mayor puntaje en la escala de Pittsburg (peor calidad del sueño), y a mayor promedio académico, menor puntaje en la escala de Pittsburg (mejor calidad del sueño). No se halló correlación entre la edad y el promedio académico con el puntaje de la escala de Epworth (tabla 4).

\section{Relación entre calidad del sueño y somnolencia diurna}

Finalmente, se encontró relación entre calidad del sueño y el grado de somnolencia diurna $\left(X^{2}=8,89\right.$, $p=0,031)$. En todos los grados de somnolencia predominó la mala calidad del sueño. Del total de las personas con SDE, el $89 \%$ tenían mala calidad del sueño, y del total de estudiantes con buena calidad del sueño, el 67,6\% presentó ligera somnolencia diurna (figura 2).

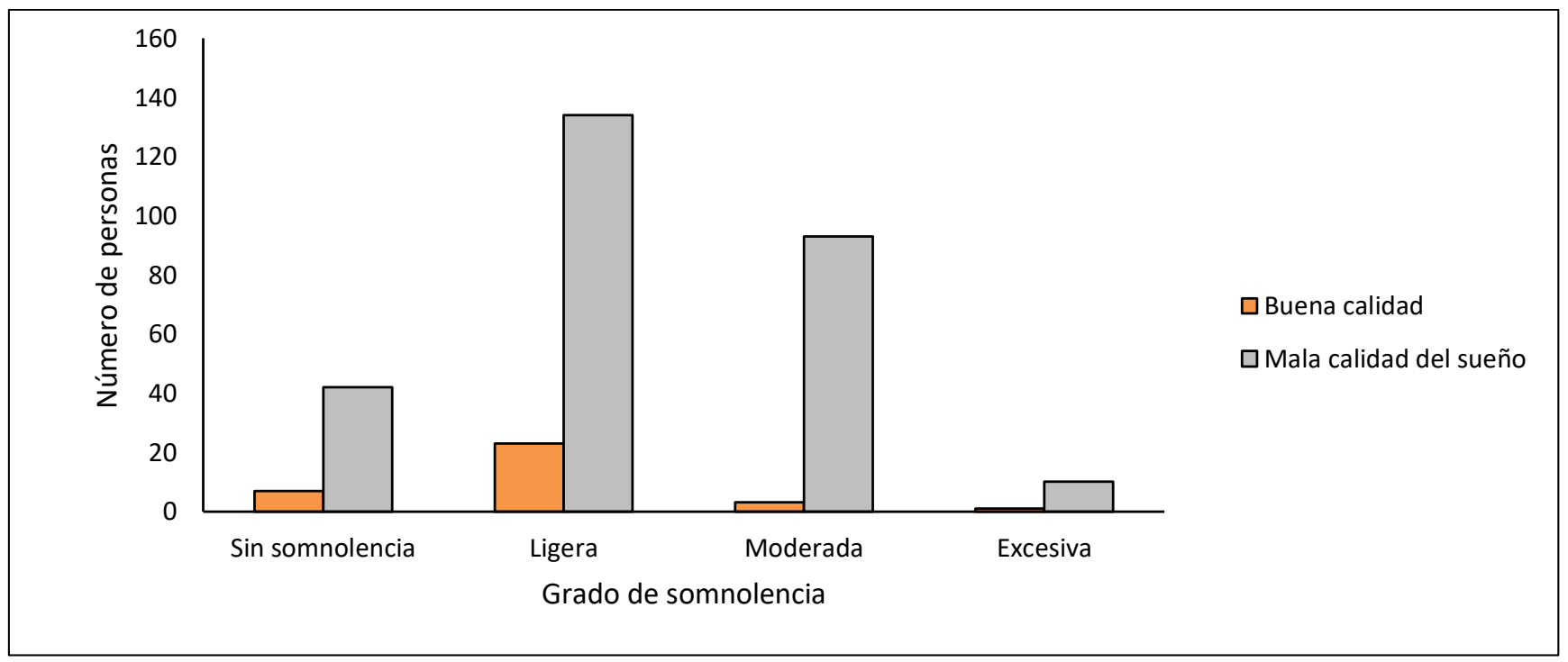

Figura 2. Grado de somnolencia diurna de los estudiantes según calidad del sueño.

\section{DISCUSIÓN}

De los 313 universitarios incluidos en el estudio, el $34 \%$ se pueden clasificar como adolescentes tardíos $(17-20 \text { años })^{13}$. Se destacan algunas características particulares como mayor participación de mujeres y de estratos socioeconómicos bajos, diferente a la investigación de Ulloque Caamaño et $a l^{3}$, que reportó mayor participación de estratos medios. Llama la atención que el 7\% refieren haber sido víctimas del conflicto armado, lo que incluye el desplazamiento forzado.

A nivel académico, similar a lo reportado por Machado-Duque et al. ${ }^{27}$ en una universidad pública del centro-occidente de Colombia, se encontró que más de la mitad $(54,3 \%)$ de los estudiantes habían perdido al menos una asignatura en su historial académico, con más frecuencia en el segundo semestre. En la Universidad del Cauca, en todos los programas de ciencias de la salud y en dicho semestre se cursan asignaturas básicas como Anatomía, Fisiología, Morfología y Genética, cuyos microcurrículos tienen altos contenidos teóricos e intensidad horaria, tanto en trabajo directo como en el independiente, lo cual podría explicar parte de este hallazgo.

En la población de estudio el 16,9\% manifestó realizar actividades extracurriculares de tipo laboral, 
inferior al 52,26\% reportado por Kaur y $\operatorname{Singh}^{28}$ en la India. Además, se identificó que la somnolencia diurna moderada o excesiva fue más frecuente en aquellos que no incluyen actividades extracurriculares de tipo laboral. No es clara la razón de esta relación, pero es posible que se generen adaptaciones a estas rutinas que involucran mayores grados de responsabilidad. Este hallazgo, sin embargo, difiere de lo identificado por Borquez ${ }^{5}$, quien reportó que incluir actividades extracurriculares favorece la aparición de trastornos del sueño. De cualquier forma, es probable que la suma de actividades de tipo laboral a los compromisos académicos implique menos tiempo de estudio efectivo; por ende, conocer esta condición desde los programas académicos podría favorecer la implementación de estrategias, programas o políticas que apoyen a los estudiantes y favorezcan la culminación exitosa de los procesos a nivel universitario.

Respecto a los hábitos, se encontró que $24,3 \%$ de los estudiantes refieren realizar actividad física cuatro veces o más a la semana, similar a investigaciones llevadas a cabo con universitarios de otras ciudades de Colombia ${ }^{29}$; sin embargo, al tratarse de áreas de ciencias de la salud, se esperaría que este tipo de prácticas fuesen más frecuentes dado el impacto conocido de la actividad física en el bienestar de las personas. Por otra parte, el consumo de tabaco fue poco frecuente entre los participantes (7,35\%), mientras que el consumo de alcohol fue referido por un $29,4 \%$, diferente a lo reportado en la investigación "Actividad física y consejería en estudiantes universitarios de primero y quinto año de medicina de Bogotá, Colombia", donde se identificó una prevalencia de consumo de tabaco que varió entre $41-53,2 \%$ y un consumo de alcohol que se situó entre $9,8-12,9 \%$ para los mismos grupos $^{30}$. Teniendo en cuenta esto, análisis posteriores podrían enfocarse en explorar las causas del consumo y las consecuencias que este puede traer dado que, si bien las cifras son bajas, son conocidos sus efectos negativos. En esa medida, se esperaría además que la universidad incremente programas de prevención y aprovechamiento del tiempo libre.
El 89,1\% de los participantes se clasificó como malos dormidores, un resultado similar al reportado para estudiantes de medicina por Monterrosa Castro et $a l^{16}$ en la Universidad de Cartagena (88,1\%), Machado-Duque et $a l^{27}$ en la Universidad Tecnológica de Pereira $(79,3 \%)$ y Escobar-Córdoba et $a l^{20}$ en la Universidad Nacional de Colombia $(79,52 \%)$. Además, se encontró que la mala calidad del sueño se relaciona con el uso de sustancias para no dormir, entre las cuales los estudiantes prefieren el café y los productos energizantes. No obstante, el uso repetido de estos se ha descrito como posible factor de riesgo para el desarrollo de enfermedades cardiacas, incluida la fibrilación auricular. Así, aunque la evidencia no es concluyente, debe tenerse en cuenta esta relación y recomendar el consumo controlado de tales productos ${ }^{31-33}$. Asimismo, a pesar de que solo un 5,8\% manifestó utilizar productos para conciliar el sueño, llama la atención que en la mayoría de los casos se automedican, por lo cual se debe hacer el seguimiento a este tipo de conductas y educar respecto a los riesgos potenciales.

En el $84,3 \%$ de los participantes se identificó algún grado de somnolencia diurna, siendo la somnolencia diurna ligera la más común $(50,1 \%)$, mientras que la SDE se manifestó en el 3,5\% de la muestra. Este hallazgo es similar al de la investigación de Machado-Duque et al. ${ }^{27}$, donde un $66,4 \%$ de participantes tuvo algún grado de somnolencia diurna, aunque en este caso la somnolencia diurna excesiva fue la más común (49,8\%). A su vez, Monsalve Barrientos et al. ${ }^{14}$, en un estudio realizado en Medellín en 2014 , señalan que el $73,5 \%$ de los participantes presentaron algún grado de somnolencia, siendo la más común la somnolencia diurna moderada con un $46,1 \%$, mientras que la somnolencia diurna excesiva fue de $10,4 \%$ del total de la muestra. Definitivamente, las altas cifras de problemas de esta índole derivan en una preocupación dado que la pérdida de sueño genera problemas en la población estudiantil, especialmente por la costumbre de disminuir el tiempo de sueño, lo cual puede traer como consecuencia déficit neurocognitivo $\mathrm{y}$, además, menor rendimiento psicomotor $3,5,16,34$. 
Steven Sánchez-Oviedo, William Esteban Solarte-Rodríguez, Sofy Thalía Correa-Carlosama, Dania Yineth Imbachí-Quinayás, Marlly Lorena Anaya-Ramírez, Andry Yasmid Mera-Mamián, Clara Inés Paz-Peña, Ángela Zúñiga-Pino

Por otra parte, se encontró correlación entre la edad y la calidad del sueño (a mayor edad, menor calidad del sueño). Investigaciones previas han descrito hallazgos similares, y se considera que los cambios evolutivos que se producen a lo largo de la vida pueden favorecer la aparición de alteraciones en el sistema regulador del ritmo sueño-vigilia ${ }^{35,36}$.

También hubo correlación débil entre el puntaje de la escala de Pittsburg y el promedio académico (a mayor calidad del sueño, mayor promedio académico). En este sentido, cabe recordar que la pérdida de sueño puede generar alteraciones como cambios del humor, depresión, incremento del estrés, abuso de sustancias como el alcohol, quejas somáticas, riesgo de accidentes vehiculares $y$ potenciales impedimentos para el aprendizaje ${ }^{1,14}$, además de irritabilidad, dificultad para concentrarse y problemas de memoria ${ }^{20}$, entre otros. Todos estos son factores que, a su vez, podrían incidir indirectamente en el rendimiento académico, tal como lo describe el estudio de Machado-Duque et $\left.a\right|^{27}$, que demostró que tener una calificación de sueño subjetiva bastante mala $(O R=2,96 ; p=0,000)$ y ser mal dormidor $(O R=3,34 ; p=0,001)$ se asociaron con el bajo rendimiento académico.

Es importante mencionar que en Colombia cada institución educativa define los criterios para calificar a los estudiantes y su rendimiento académico. En el caso de la Universidad del Cauca, este se refleja por el promedio de las calificaciones obtenidas a lo largo de cada semestre y según las actividades que se realizan en cada asignatura. Así, la nota definitiva de una asignatura es "la nota final o la que se obtiene en examen de habilitación o de validación", y esta se considera aprobada cuando "es igual o superior a tres puntos cero $(3,0)$ " y perdida en el caso contrario. En ese orden de ideas, se considera bajo rendimiento académico si el estudiante ha perdido alguna asignatura que curse en calidad de repitente por primera vez ${ }^{37}$, pero en pocas ocasiones esta calificación tiene en cuenta el proceso o el nivel de alcance de los objetivos en una asignatura.

Respecto a las limitaciones de la presente investigación, se reconoce que la muestra no es representativa de la totalidad de estudiantes de la Facultad de Ciencias de la Salud, dado que la participación fue voluntaria y la representación de los programas no fue proporcional. Adicionalmente, algunos de los datos de la caracterización sociodemográfica y académica no fueron diligenciados de forma completa, lo que generó información faltante, y otras variables, especialmente relacionadas con tiempo dedicado a actividades teóricas y prácticas, debieron ser eliminadas por el alto porcentaje de errores a pesar de que durante el pilotaje no se identificaron este tipo de dificultades.

Entre las fortalezas se destaca que aproximadamente el $35 \%$ de la totalidad de los estudiantes de la Facultad de Ciencias de la Salud participó a pesar de ser un formulario en línea con un total de 69 ítems. Además, los instrumentos utilizados para evaluar tanto la calidad del sueño como la somnolencia diurna han sido validados y ampliamente utilizados en la población universitaria, lo cual favorece la calidad de la medición. Finalmente, se resalta que es uno de los primeros estudios en calidad del sueño que involucra diferentes programas de ciencias de la salud en general, dado que la mayoría de las investigaciones previas se han enfocado en los programas de Medicina y Enfermería.

A partir de los resultados, surgen como recomendaciones educar en la higiene del sueño a los estudiantes de la Facultad de Ciencias de la Salud, explorar las causas de la inactividad física, y motivar la realización de ejercicio desde áreas como bienestar y cultura, al tiempo que, por parte de los estudiantes, se sugiere aprovechar los espacios asignados para la práctica de diferentes deportes. Por último, se considera importante proyectar desde la universidad una evaluación más integral del rendimiento académico de un estudiante que pueda incluir, además de las calificaciones, los logros en términos de las competencias alcanzadas, las cuales podrían ser valoradas por aspectos como la participación en eventos académicos, el emprendimiento, el liderazgo o la proyección social.

En suma, con estos hallazgos se pretende generar información que pueda ser utilizada en las políticas de bienestar estudiantil de la universidad, además de suministrar datos que pueden ser de utilidad en investigaciones posteriores. 


\section{AGRADECIMIENTOS}

Los autores agradecen al fisioterapeuta Raúl Orozco por su apoyo en la recolección de información, a los estudiantes de los diferentes programas por su participación en el diligenciamiento de la encuesta y a la Universidad del Cauca por su compromiso con los procesos de investigación.

\section{DECLARACIÓN SOBRE CONFLICTO DE INTERESES}

Como autores declaramos no tener conflicto de intereses.

\section{CONTRIBUCIÓN DE LOS AUTORES}

Primer autor: planteamiento de la propuesta, generación del protocolo, recolección de información, discusión de resultados.

Segundo autor: planteamiento de la propuesta, generación del protocolo, recolección de información, discusión de resultados.

Tercer autor: recolección de información, análisis de datos, discusión de resultados.

Cuarto autor: recolección de información, análisis de datos, discusión de resultados.

Quinto autor: recolección de información, análisis de datos, discusión de resultados.

Sexto autor: diseño metodológico, análisis de datos, discusión de los resultados y edición de versión final por ser publicada.

Séptimo autor: diseño metodológico, análisis de datos, discusión de los resultados y edición de versión final por ser publicada.

Octavo autor: diseño metodológico, análisis de datos, discusión de los resultados y edición de versión final por ser publicada.

\section{REFERENCIAS BIBLIOGRÁFICAS}

1. Rosales Mayor E, Rey De Castro Mujica J. Somnolencia: Qué es, qué la causa y cómo se mide. Acta Médica Peruana [revista en la Internet]. 2010; 27(2): 137-43. Disponible en: http://www.scielo.org.pe/scielo.php?script=sci_abs
tract\&pid=S1728-

59172010000200010\&lng=es\&nrm=iso\&tlng=es

2. Siegel JM. Do all animals sleep? Trends Neurosci. 2008; 31(4): 208-13. Disponible en: https://www.sciencedirect.com/science/article/pii/ S0166223608000623?casa_token=KHimRXDaTUkA AAAA:G2_Vg8BMujenz2itd61GfVbG0COeH6SDsctdQNnZmLhrtnsnNDuGOAjRZ1Imdiuvcm2Gp_yJM

3. Ulloque-Caamaño L, Monterrosa-Castro Á, Carriazo-Julio S. Somnolencia diurna y rendimiento académico de estudiantes de medicina de una universidad pública colombiana. Revista Ciencias Biomédicas [revista en la Internet]. 2013; 4(1): 11. Disponible en: http://grupodeinvestigacionsaluddelamujer.com.co /PDF/Femsalud/ARTICULOS_CIENTIFICOS/4_SOMN OLENCIA\%20DIURNA\%20EN\%20ESTUDIANTES\%20 DE\%20MEDICINA.pdf

4. Barrechenea Loo MB, Gómez Ceballos C, Huaira Peña AJ, Pregúntegui Loayza I, Aguirre Gonzales M, De Castro Mujica JR. Calidad de sueño y excesiva somnolencia diurna en estudiantes del tercer $y$ cuarto año de Medicina. Ciencia e Investigación Médico Estudiantil Latinoamericana (CIMEL) [revista en la Internet]. 2010; 15(2): 54-8. Disponible en: https://www.cimel.felsocem.net/index.php/CIMEL/ article/view/117

5. Borquez P. Calidad de sueño, excesiva somnolencia diurna y salud autopercibida en estudiantes universitarios. Eureka [revista en la Internet]. 2011; 8(1): 81-91. Disponible en: http://pepsic.bvsalud.org/scielo.php?script=sci_art text\&pid=S2220-90262011000100009

6. Guerra Pozo M. Trastornos del sueño [Internet]. Universidad de Sevilla; 2018. Disponible en: https://idus.us.es/bitstream/handle/11441/82818/ TFG\%20MIRELLA\%20GUERRA\%20POZO.pdf?seque nce $=1 \&$ isAllowed $=y$

7. Burbano Obando JP, Sánchez Sánchez JP, Mera Mamián AY. Biomarcadores de estrés laboral en residentes: artículo de revisión. Revista de la Facultad de Ciencias de la Salud Universidad del 
Steven Sánchez-Oviedo, William Esteban Solarte-Rodríguez, Sofy Thalía Correa-Carlosama, Dania Yineth Imbachí-Quinayás, Marlly Lorena Anaya-Ramírez, Andry Yasmid Mera-Mamián, Clara Inés Paz-Peña, Ángela Zúñiga-Pino

Cauca [revista en la Internet]. 2019; 21(2): 24-31.

Disponible

en:

https://revistas.unicauca.edu.co/index.php/rfcs/art icle/view/1373

8. Mendoza LA, Caballero S, Ormea V, Aquino R, Yaya E, Portugal A, et al. Neurociencia del sueño: rol en los procesos de aprendizaje y calidad de vida. Apuntes de Ciencia \& Sociedad [revista en la Internet]. 2017; 7(2): 103-9. Disponible en: http://journals.continental.edu.pe/index.php/apun tes/article/view/524

9. Durán Agüero SA, Rosales Soto G, García Milla P, Moya Cantillana C. Insomnio, latencia al sueño, y cantidad de sueño en estudiantes universitarios chilenos durante el periodo de clases y exámenes. Revista Científica Salud Uninorte [revista en la Internet]. 2017; 33(2): 75-85. Disponible en: http://rcientificas.uninorte.edu.co/index.php/salud /article/view/9448

10. Vilchez-Cornejo J, Quiñones-Laveriano D, FailocRojas V, Acevedo-Villar T, Larico-Calla G, MucchingToscano $S$, et al. Salud mental y calidad de sueño en estudiantes de ocho facultades de medicina humana del Perú. Revista Chilena de Neuro-psiquiatría [revista en la Internet]. 2016; 54(4): 272-81. Disponible

en:

https://scielo.conicyt.cl/scielo.php?script=sci_abstr act\&pid=S0717-

$92272016000400002 \& \operatorname{lng}=e s \& n r m=i s o \&$ tlng=es

11. Arboledas GP, Campos MS. Fisiología del sueño y sus trastornos. Ontogenia y evolución del sueño a lo largo de la etapa pediátrica. Relación del sueño con la alimentación. Clasificación de los problemas y trastornos del sueño. Pediatría Integral [revista en la Internet]. 2018; XXI(8): 358-71. Disponible en: https://www.pediatriaintegral.es/publicacion2018-12/fisiologia-del-sueno-y-sus-trastornosontogenia-y-evolucion-del-sueno-a-lo-largo-de-laetapa-pediatrica-relacion-del-sueno-con-laalimentacion-clasificacion-de-los-problemas-ytrastornos-del-sueno

12. Niño García JA, Barragán Vergel MF, Ortiz Labrador JA, Ochoa Vera ME, González Olaya HL. Factores asociados con somnolencia diurna excesiva en estudiantes de Medicina de una institución de educación superior de Bucaramanga. Revista Colombiana de Psiquiatría [revista en la Internet]. 2019; 48(4): 222-31. Disponible en: http://www.elsevier.es/es-revista-revistacolombiana-psiquiatria-379-articulo-factoresasociados-con-somnolencia-diurnaS0034745018300209

13. Talero C, Durán F, Pérez I. Sueño: características generales. Patrones fisiológicos y fisiopatológicos en la adolescencia. Revista Ciencias de la Salud [revista en la Internet]. 2013; 11(3): 333-48. Disponible en: https://www.redalyc.org/articulo.oa?id=56229183 008

14. Monsalve Barrientos K, García Arango SP, Chávez Bertel FD, David Marriaga A, Cardona Arias JA. Prevalencia de insomnio y somnolencia en estudiantes de medicina pertenecientes a una institución educativa de Medellín (Colombia), 2013. Archivos de Medicina [revista en la Internet]. 2014; 14(1): $\quad$ 92-102. Disponible en: https://www.redalyc.org/articulo.oa?id=27383216 4009

15. Carrillo-Mora P, Barajas-Martínez KG, SánchezVázquez I, Rangel-Caballero MF, Carrillo-Mora $P$, Barajas-Martínez KG, et al. Trastornos del sueño: ¿qué son y cuáles son sus consecuencias? Revista de la Facultad de Medicina (México) [revista en la Internet]. 2018; 61(1): 6-20. Disponible en: http://www.scielo.org.mx/scielo.php?script=sci_ab stract\&pid=S0026-

$17422018000100006 \& \operatorname{lng}=e s \& n r m=i s o \&$ tlng=es

16. Monterrosa Castro A, Caamaño LU, Carriazo J. Calidad del dormir, insomnio y rendimiento académico en estudiantes de medicina. Duazary [revista en la Internet]. 2014; 11(2): 85-97. Disponible en: https://revistas.unimagdalena.edu.co/index.php/d uazary/article/view/814

17. Vales L, Mora B, Martínez J, Gómez C, Lungo R, Fígoli I. Niveles de impulsividad motora asociados a somnolencia diurna excesiva en niños y niñas en situación de vulnerabilidad social. Cuadernos de Neuropsicología. Panamerican Journal of Neuropsychology [revista en la Internet]. 2018; 12(2). Disponible en: 
https://www.cnps.cl/index.php/cnps/article/view/ 337

18. Guerra Sotomayor EM, Menéndez Vinces WA. Asociación entre el nivel de calidad de sueño, somnolencia y calidad de vida del personal de salud que trabaja y realiza turnos en un Hospital de II Nivel de la provincia de Esmeraldas - Ecuador, durante el periodo enero - marzo 2019 [Internet]. Universidad Católica del Ecuador; 2019. Disponible en: http://repositorio.puce.edu.ec/handle/22000/1675 5

19. Maya $S$ de la $P$, Lubert $C D$, Londoño $D M M$, Chaurra JT, Osorio LSN. Calidad de sueño y somnolencia diurna excesiva en estudiantes universitarios de diferentes dominios. Hacia la Promoción de la Salud [revista en la Internet]. 2019; 24(1): 84-96. Disponible en: https://revistasojs.ucaldas.edu.co/index.php/hacial apromociondelasalud/article/view/3592

20. Escobar-Córdoba $F$, Benavides-Gélves RE, Montenegro-Duarte HG, Schmalbach JHE. Somnolencia diurna excesiva en estudiantes de noveno semestre de Medicina de la Universidad Nacional de Colombia. Revista Facultad de Medicina [revista en la Internet]. 2011; 59(3): 191-200. Disponible

en: https://revistas.unal.edu.co/index.php/revfacmed/ article/view/26453

21. Casanova Barrios C, Quintana Honores M, Álvarez Niño L. Estilos de Vida y su implicación en la salud de los estudiantes Universitarios de las Ciencias de la Salud: Una revisión sistemática. Revista Científica de la UCES [revista en la Internet]. 2018; 23(2): 98-126. Disponible en: http://dspace.uces.edu.ar:8180/xmlui/handle/1234 56789/4520

22. García López V. El trabajo y los otros efectos letales de la falta de sueño. Anales del Sistema Sanitario de Navarra [revista en la Internet]. 2019; 42(3): 357-9. Disponible en: https://scielo.isciii.es/pdf/asisna/v42n3/11376627-asisna-42-03-357.pdf
23. Escobar-Córdoba F, Eslava Schmalbach J. Validación colombiana del índice de calidad de sueño de Pittsburgh: Neurología.com. Revista de Neurología [revista en la Internet]. 2005; 40(3): 150$5 . \quad$ Disponible en: http://www.neurologia.com/articulo/2004320

24. Chero Pisfil SL. Somnolencia diurna y su relación con la inteligencia emocional en estudiantes universitarios de terapia física de una universidad de Lima, 2018. Tesis de Maestría en Educación [Internet]. Universidad de Lima; 2018. Disponible en:

https://repositorio.ucv.edu.pe/handle/20.500.1269 2/20049

25. The Jamovi Project. Jamovi (Versión 1.2) [Internet]. 2020. Disponible en: https://www.jamovi.org

26. R Core Team. R: A Language and environment for statistical computing (Versión 3.6) [Internet]. Computer software. 2019. Disponible en: https://cran.r-project.org

27. Machado-Duque ME, Echeverri Chabur JE, Machado-Alba JE. Somnolencia diurna excesiva, mala calidad del sueño y bajo rendimiento académico en estudiantes de Medicina. Revista Colombiana de Psiquiatría [revista en la Internet]. 2015; 44(3): 137-42. Disponible en: http://www.elsevier.es/es-revista-revista-

colombiana-psiquiatria-379-articulo-somnolenciadiurna-excesiva-mala-calidad-S0034745015000426

28. Kaur G, Singh A. Excessive daytime sleepiness and its pattern among Indian college students. Sleep Med [revista en la Internet]. 2017; 29: 23-8. Disponible en: https://pubmed.ncbi.nlm.nih.gov/28153211

29. Miranda González $Y$, Rodríguez Puello $M$, Freile Molinares B, Peña Molina AM. Niveles de actividad física en los estudiantes de la facultad de ciencias de la salud de la Universidad Simón Bolívar de Barranquilla: estudio transversal. Salud en Movimiento [revista en la Internet]. 2015; 7(1): 22$8 . \quad$ Disponible en: 
Steven Sánchez-Oviedo, William Esteban Solarte-Rodríguez, Sofy Thalía Correa-Carlosama, Dania Yineth Imbachí-Quinayás, Marlly Lorena Anaya-Ramírez, Andry Yasmid Mera-Mamián, Clara Inés Paz-Peña, Ángela Zúñiga-Pino

https://bonga.unisimon.edu.co/handle/20.500.124 $42 / 1366$

30. Tovar G, Rodríguez Á, García G, Tovar JR. Actividad física y consejería en estudiantes universitarios de primero y quinto año de medicina de Bogotá, Colombia. Revista Universitaria de Salud [revista en la Internet]. 2016; 18(1): 16. Disponible en:

http://revistas.udenar.edu.co/index.php/usalud/ar ticle/view/2719

31. Manrique $\mathrm{Cl}$, Arroyave-Hoyos CL, Galvis-Pareja D. Bebidas cafeinadas energizantes: efectos neurológicos y cardiovasculares. latreia [revista en la Internet]. 2018; 31(1): 65-75. Disponible en: https://revistas.udea.edu.co/index.php/iatreia/arti cle/view/326194

32. González Estévez M, Rivadeneyra Espinoza L, Robles-Carrillo CC, Soto-Vega E, Ceja-Ramírez LG. Efectos de las bebidas con cafeína en la calidad de sueño en alumnos de Medicina en Puebla. Ciencia e Investigación Médico Estudiantil Latinoamericana (CIMEL) [revista en la Internet]. 2017; 22(2): 30-4. Disponible

en: https://www.cimel.felsocem.net/index.php/CIMEL/ article/view/638

33. Fon Tejada MC. Consumo de bebidas energizantes como factor asociado a la corta duración de sueño en estudiantes universitarios. Trujillo, Perú. Universidad privada Antenor Orrego; $2018 . \quad$ Disponible en: http://200.62.226.186/handle/20.500.12759/4289

34. Caicedo Ochoa Y, Urrutia Gómez A, Sánchez Fonseca S, Rodríguez Chaves D. Prevalencia del grado de somnolencia y su relación con el rendimiento académico en estudiantes de medicina de la ciudad de Tunja, Colombia, durante el año 2014. Ciencia e Investigación Médico Estudiantil Latinoamericana (CIMEL) [revista en la Internet]. 2015; 20(2): 58-62. Disponible en: https://www.cimel.felsocem.net/index.php/CIMEL/ article/view/584

35. Madrid-Valero JJ, Martínez-Selva JM, Ribeiro do Couto B, Sánchez-Romera JF, Ordoñana JR. Age and gender effects on the prevalence of poor sleep quality in the adult population. Gaceta Sanitaria [revista en la Internet]. 2017; 31(1): 18-22. Disponible

en: https://www.sciencedirect.com/science/article/pii/ S0213911116301182

36. Lira D, Custodio N. Los trastornos del sueño y su compleja relación con las funciones cognitivas. Revista de Neuro-Psiquiatría [revista en la Internet]. 2018; 81(1): 20-8. Disponible en: http://www.scielo.org.pe/scielo.php?script=sci_abs tract\&pid=S0034-

$85972018000100004 \& \operatorname{lng}=e s \& n r m=i s o \& \operatorname{tn} g=e n$

37. Universidad del Cauca. Acuerdo No. 002 de 1988 [Internet]. Popayán, Cauca. Disponible en: http://portal.unicauca.edu.co/versionP/documento s/acuerdos/acuerdo-no-002-de-1988-por-el-cualse-expide-el-reglamento-estudiantil-de-launiversidad-del-caucadomayo 